\title{
GLANDECTOMY WITH PRESERVATION OF CORPORA CAVERNOSA IN THE TREATMENT OF PENILE CARCINOMA
}

\author{
ALUIZIO G. DA FONSECA, GILFLÁVIO N. RABELO, KALLENE S.M. VIDAL, FERNANDO \\ J. DE SOUSA
}

Ofir Loyola Hospital, Belém, Pará, Brazil

\begin{abstract}
Introduction: The objective of this work is to describe a conservative surgical technique as an alternative to classic penile amputations, aiming the local control of the disease, in addition to trying to preserve the patient's sexual function.

Surgical technique: After a circular incision of the skin around the penis, the subfascial plane is developed until the base of the organ. The dorsal neurovascular bundle and the urethra are isolated in their distal extremities. The neurovascular bundle is sectioned distally. A retrocoronal dissection plane is developed between the glans and the corpora cavernosa. When this stage is complete, the glans is fixed only to the urethra, which is distally sectioned as well. The neurovascular bundle is fixed to the dorsal albuginea. Following the spatulation of the urethra, a neomeatus is created using the overlay skin of the penis. Between January 2001 and July 2002, we employed this technique in 6 patients who had epidermoid carcinoma of the penis, which were limited to the glans, superficial, well or moderately differentiated and measuring up to $3 \mathrm{~cm}$.

Comments: Several conservative surgical methods for treatment of carcinoma of the penis aim the organ's preservation, in an attempt of improving the quality of life of patients, however the indexes of local recurrence and failure in disease control are significant. The described technique showed to be safe and effective for disease control, in addition to preserving sexual function in all patients who were treated, representing, thus, a quite appealing conservative surgical alternative in selected cases.
\end{abstract}

Key words: penis; penile cancer; surgery; surgical procedures, minimally invasive

Int Braz J Urol. 2003; 29: 437-40

\section{INTRODUCTION}

The epidermoid carcinoma of the penis is a rare neoplasia in developed countries, however in underdeveloped countries its frequency reaches high levels. In the state of Pará, Brazil, its incidence reaches significant indexes, being the second most frequent urogenital tumor in our institution. Surgical treatment is the safest method for management and aims to resect the primary lesion with safe margins. Penile amputations are still the "gold standard" in the disease's surgical treatment; nevertheless repercussions in the psychic and social sphere of patients are devastating (1). The introduction of conservative surgical methods, which aim to completely excise the primary tumor, respecting proper surgical margins and, at the same time, preserving the organ's shape and function, tried to reduce such consequences (2). This work describes a surgical alternative to partial amputation: glandectomy with preservation of the corpora cavernosa, which can be indicated in small, non-infiltrating penile tumors that are limited to the glans (Figure-1). 


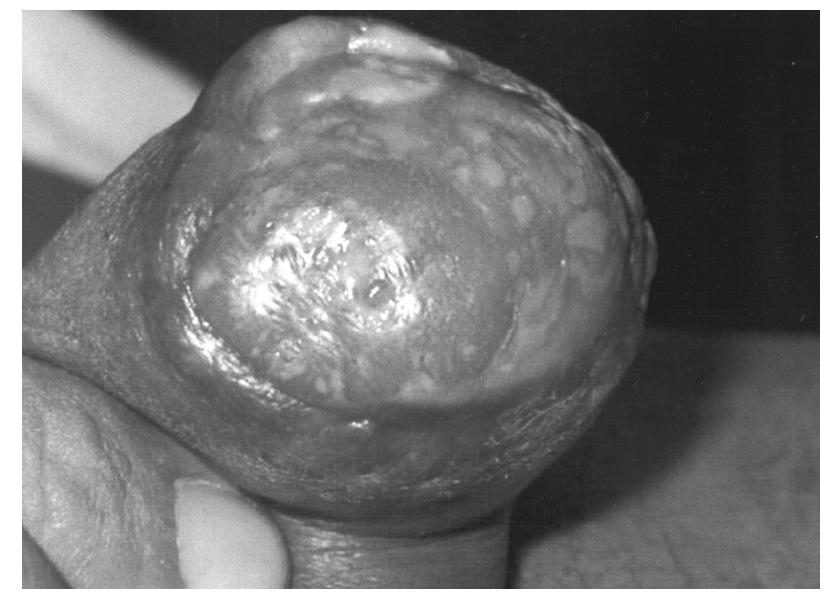

Figure 1 - Epidermoid carcinoma limited to the glans penis, with approximately $2 \mathrm{~cm}$ in length.

\section{SURGICAL TECHNIQUE}

Patients undergo an enema in the night previous to surgery and receive broad-spectrum antibiotic (intravenous cefazolin, $1 \mathrm{~g}$ ) after the anesthetic induction, which is maintained until the second postoperative day, when it is replaced by an oral antibiotic.

Under spinal anesthetic block, the patient is placed in horizontal dorsal decubitus. The procedure begins with a circular incision of the skin, around the penis, deepening until the Buck's fascia. At this point,

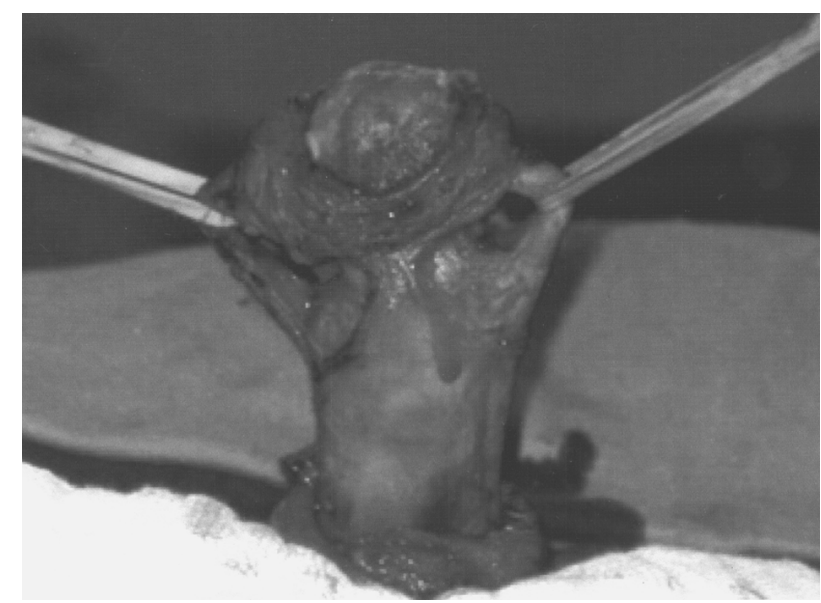

Figure 2 - Dissection of dorsal neurovascular bundle and urethra.

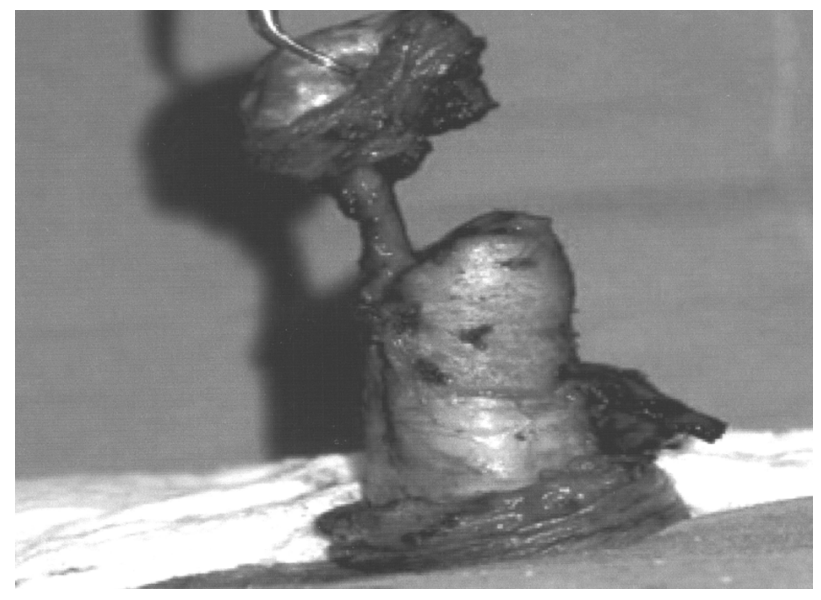

Figure 3 - After sectioning the neurovascular bundle and dissecting a subcoronal plane, the glans remains fixed only to the urethra.

the subfascial plane is developed until the base of the penis, unwrapping the organ. Next, the isolation and repair of the dorsal neurovascular bundle and the urethra are performed, in their distal extremities (Figure-2). The neurovascular bundle is distally sectioned as in the classic partial amputation. A retro-coronal dissection plane is developed under the neurovascular bundle, between the glans and the extremities of the corpora cavernosa, with complete preservation of the latter. Upon completing this surgical time, the glans, completely dissected, is fixed only to the urethra, which is also distally sectioned (Figure-3).

A careful inspection of the lesion bed is performed relative to the extremities of the corpora cavernosa, where biopsies are made for freezing histological analysis. Following the fixation of the neurovascular bundle to the albuginea in the dorsal surface of the penis, with separate stitches, a neomeatus is then created with the spatulate distal urethra, using the overlay skin of the penis, previously dissected (Figure-4). A 16F Foley catheter is maintained inside the urethra for urinary drainage during 72 hours.

\section{COMMENTS}

The surgical treatment for cancer of the penis, concerning the primary lesion, has been based, for many years, in total or partial penile amputations, 


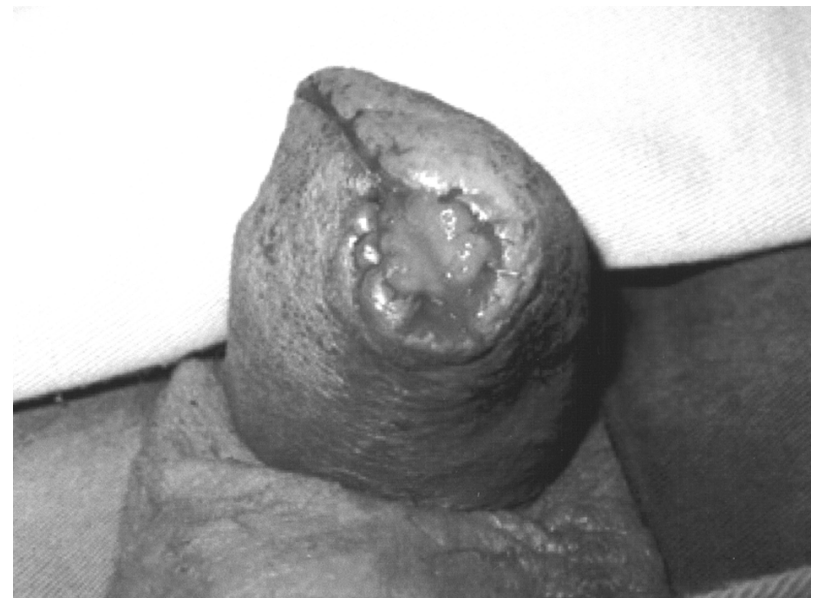

Figure 4-Creation of the neomeatus using the overlay skin of the penis.

which preconize margins between 15 and $30 \mathrm{~mm}$ from the lesion (2). Since the vast majority of penile tumors originate in the glans, coronal sulcus or foreskin, partial amputations are frequently employed. Such margins optimize the possibility of eradicating microscopic foci beyond the macroscopic limits of the tumor, but significantly reduce the penile length (1). The limitations to sexual intercourse and masturbation are obvious in cases of total amputations. However, in patients submitted to partial amputations, despite the reduction in the penile length, vaginal penetration is frequently possible, as well as orgasm and ejaculation (2).

A few works in literature assess the consequences of these procedures in psychological, social and sexual spheres of treated patients. Jensen (1), in a prospective analysis of 119 patients submitted to partial amputation, observed that $90 \%$ of patients with residual penile stump larger than $6 \mathrm{~cm}$ maintained a good sexual function, which occurred in $45 \%$ of those with a stump between 4 and $6 \mathrm{~cm}$ and, in only $25 \%$ of those who presented stumps between 2 and $4 \mathrm{~cm}$.

An analysis of the literature demonstrates that the local recurrence indexes in classic partial amputations range from 0 to $20 \%$, showing a quite satisfactory control of the primary lesion with this method. However, the 5-year survival depends mainly on the occurrence of metastases in regional lymph nodes, which in turn, is dependant on other prognostic factors, and therefore, there is the possibility of regional recurrence without local relapse of the disease (3). Such behavior is explained by the pattern of lymphatic dissemination, which occurs by embolization. For this reason, it is possible to use more conservative procedures for surgical control of the primary lesion in selected cases (2).

The definition of conservative surgery comprises every procedure that aims the complete excision of the primary tumor, respecting proper surgical margins and, at the same time, preserving the organ's shape and function $(2,4)$. Among these, stand out local excision, circumcision, micrographic surgery, laser surgery and other recent conservative surgical trends (5).

The concern with quality of life has led many authors to propose technical modifications for classic amputations. The reduction in classic limits seems to be a new emerging concept. Agrawal et al., demonstrate that $10 \mathrm{~mm}$ margins are enough for grade I or II tumors, however in undifferentiated tumors, 15 mm margins offer higher safety (4).

Local excision of the primary lesion, in cancer of the penis, presents local recurrence indexes that reach $100 \%(2,4)$. Circumcision for treatment of lesions located in the foreskin demonstrates recurrence in up to $50 \%$ of cases (2). In a study conducted by Bissada (2), micrographic surgery demonstrated local recidivation in $8 \%$ of the patients and a 5-year survival of $68 \%$, among these, 6 patients with undifferentiated tumors who evolved to death due to uncontrolled metastases (2). The use of laser in its various modalities shows a local recurrence that ranges between 4 and $33 \%$ and, survival at the end of follow-up between 94 and 100\%. It is worth to point out that the follow-up in these studies is short, and additionally the local recurrence indexes with the method in undifferentiated lesions reaches $100 \%$, according to some series (4).

Pizzocaro et al., compared the results from 75 patients with T1N0 lesions conservatively treated, using radiotherapy, circumcision or ablative laser surgery associated with systemic chemotherapy with 26 patients submitted to classic amputations. In the group treated with conservative methods 15 presented local recurrence, however, with salvage surgery, only one patient died from the disease. The failure indexes 
regarding the control of lymph node disease, with or without local recurrence, were similar in the 2 groups (5).

Davis et al., described the use of glandectomy, with preservation of the corpora cavernosa, in a patient who had an undifferentiated epidermoid carcinoma, located in the glans, who remained free of recurrence during 22 months of follow-up. This patient maintained a good capacity for sexual intercourse, according to the authors (3). Motivated by this report, we used the technique in 6 patients with histological diagnosis of epidermoid carcinoma of the penis, well or moderately differentiated, superficial, localized in the glans and with a lesion measuring up to $3 \mathrm{~cm}$ in length. Highly satisfactory results were obtained from the cosmetic, functional and oncologic point-of-view. All patients presented negative surgical margins in pathologic analysis and maintained a quite satisfactory sexual function. There was no local recurrence, despite the quite limited follow-up, which ranged from 6 to 18 months. Surgical complications were not observed.

The highly satisfactory initial results concerning safety, efficacy and conservation of penile length, make the glandectomy with cavernous preservation a surgical alternative for patients with pre-malignant lesions, extensive "in situ" carcinoma, verrucous carcinoma and well differentiated, superficial $\mathrm{T} 1$ tumors that are limited to the glans and measure up to $3 \mathrm{~cm}$.

\section{REFERENCES}

1. Jensen MS: Cancer of the penis in Denmark 1942 to 1962 (511 cases). Danish Med Bull. 1977; 24: 66-72.

2. Bissada NK: Conservative extirpative treatment of cancer of the penis. Urol Clin North Am. 1992; 19: 283-90.

3. Davis JW, Schellhammer PF, Schlossberg SM: Conservative surgical therapy for penile and urethral carcinoma. Urology. 1999; 53: 386-92.

4. Agrawal A, Pai D, Ananthakrishnan N, Smile SR, Ratnakar C: The histological extent of local spread of carcinoma of the penis and its therapeutic implications. BJU Int. 2000; 85: 299-301.

5. Pizzocaro G, Stagni S, Di Palo A: Conservative treatment possibilities in category T1N0 squamous cell carcinoma (SCC) of the penis. J Urol. 1997; 157 (suppl): 47.

\author{
Correspondence address: \\ Dr. Aluízio Gonçalves da Fonseca \\ Travessa Dom Romualdo de Seixas, 1344 \\ Belém, PA, 66055-200, Brazil \\ Fax: + 5591 241-5988 \\ E-mail: agf@supridad.com.br
}

